\title{
Altersgedanken zu Tod und Sterben
}

\section{Luc Ciompi*}

* Prof. Dr. med. em., vorm Direktor der Sozialpsychiatrischen Universitätsklinik Bern

Korrespondenz:

Prof. Dr. med. em. Luc Ciompi Route de la Cita 6

CH-1092 Belmont-sur-Lausanne

cioluci@freesurf.ch

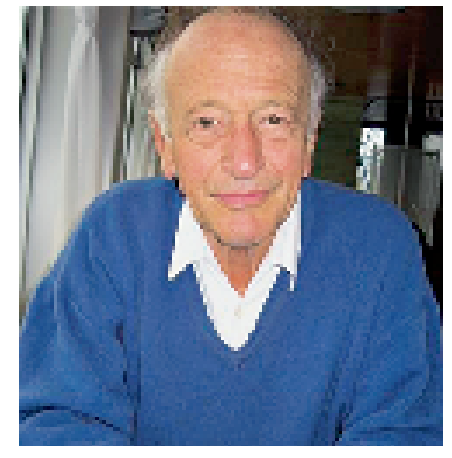

Luc Ciompi

Vielerlei Gründe bewegen mich dazu, über das Thema Tod und Sterben nachzudenken. Schon meines eigenen Alters wegen - ich bin 77 - ist es vorder- oder hintergründig allgegenwärtig. Auch als Arzt und Psychiater war ich damit immer wieder konfrontiert. Beruflich und privat erlebte Lebens- und Sterbensgeschichten fügen sich im Lauf eines Arztlebens zu einem Bild von verwirrender Vielfalt. Wie soll man Fragen von Jungen beantworten, wie «es» nun eigentlich sei im Alter? Altersprobleme haben mich - u.a. im Rahmen von Untersuchungen zur Innenwelt von Alzheimerpatienten - auch schon als junger Forscher interessiert. Ausserdem schrieb ich einmal einen Leserbrief zum Thema der ärztlichen Sterbehilfe [1], der mir einen Rattenschwanz von Zuschriften, Diskussionen und, vor allem, von eigenen und zum Teil recht queren Folgegedanken eintrug. Diese in all ihrer Widersprüchlichkeit den Lesern dieser Zeitung zu unterbreiten ist das Ziel der nachfolgenden Zeilen.

Vorauszuschicken ist, dass Gedanken zu Tod und Sterben wohl kaum anders als stark subjektiv gefärbt sein können. Dennoch möchte ich mit einem Aspekt beginnen, der mir recht objektiv vorkommt, nämlich mit dem Tod aus evolutionärer Sicht.

\section{Gottlob können wir sterben!}

Evolutionär gesehen gilt der Tod als überaus sinnvolle und geradezu geniale Erfindung: Erst das Verschwinden des Alten ermöglicht, bei aller genetischen Fortdauer des Bewährten, die Anpassung eines immer wieder jungen Lebens an eine ständig sich verändernde Umwelt. Man stelle sich bloss vor, wie verheerend die Situation wäre, wenn wir unsterblich wären: Alle Alten und alles Alte immer noch da und im Weg - nicht nur
Hitler, Sokrates, Nero, Goethe, sondern auch die ganz gewöhnliche Masse von Menschen, immer grösser und erdrückender: eine schreckliche Vision! Wie wir die Sache auch drehen und wenden: Es ist sehr gut, dass wir alle sterben können - und folglich ist es auch ganz in Ordnung, dass ich selbst bald einmal sterben werde.

\section{Glücklicherweise leben wir nicht zweihundert Jahre!}

Hier freilich beginnen die Widersprüche: Ich möchte doch gerne so lange und gut wie möglich leben. Auch dieser Wille zum Leben um jeden Preis ist gewiss sinnvoll und evolutionär verankert. Genauso sinnvoll sind indes die dem individuellen Lebenswillen von der Natur gesetzten Grenzen. Mit anderen Worten: Auch den ewigen Menschheitstraum von der Langlebigkeit über jedes «biblische Mass» hinaus halte ich für eine gefährliche Illusion. Schon die auf 70-80 Jahre verdoppelte durchschnittliche Lebenserwartung stellt bekanntlich zunehmend grosse demographische, psychologische und nicht zuletzt ökonomische Probleme. Wie soll das erst werden, wenn die Lebensdauer einmal auf 120 , 150 oder gar 200 Jahre verlängert werden kann? - Wiederum: verheerend in fast jeder Hinsicht, entgegen allen unrealistischen Heilserwartungen, die uns eine fortschrittsgläubige Science fiction vorgaukeln will.

\section{Der «Skandal des Todes» und die angebliche Angst vor dem Nichts}

Doch lassen wir die ungewisse Zukunft, und wenden wir uns der Realität zu: Der gewisse Tod und damit das «Verschwinden ins Nichts» sei ein unerträglicher Skandal, soll sogar der eingefleischte Atheist und Existentialist Jean-Paul Sartre reklamiert haben. Aber was ist eigentlich an diesem Verschwinden so furchtbar? Macht nicht gerade der Tod das Leben zu einem ungeheuer wertvollen Geschenk? Soll ich für dieses Geschenk denn nicht nur danken, sondern es auch noch auf ewig festhalten wollen - über alle Spuren hinaus, die noch der Geringste unter uns obligat hinterlässt? Denn im geistigen Sinn geht ja nichts, gar nichts je völlig verloren: Was immer ich war und tat, im guten wie im bösen, wirft eine Zeitlang seine kleinen oder grösseren Wellen, die alle früher oder später in die anonyme Solitärwelle des Gesamtgeschehens einmünden. - Ist das denn 
nicht genug? Meines Erachtens langt dies vollkommen; ich möchte keine ewige Bleibe noch Jugend (um Gottes willen!), und vor dem Eingehen in solch ein «Nichts» verspüre ich keinerlei Angst.

\section{Lob des Alters}

«Wer das Alter lobt, der hat ihm nicht ins Antlitz geschaut», zitierte mein geschätzter Berufskollege Paul Parin neulich in einem NZZ-Interview [2] den italienischen Philosophen Norberto Bobbio. - Es stimmt natürlich, dass das Alter unausweichlich mit Beschwerden und Verlusten bis hin zum Tod einhergeht. Wohl am schwersten zu ertragen sind Krankheit, Schmerz und Vereinsamung. Aber ich kenne nicht wenige Menschen, mit Einschluss meiner selbst, die das Alter über weite Strecken auch als eine faszinierend reiche Zeit voller Neuheiten und Überraschungen erleben. Was allein doch schon die - vielen vergönnte - Grosselternrolle mit sich bringt an ungeahnten Möglichkeiten des Lebens und Liebens! Nicht unähnlich ist die Rolle, die man als offizieller oder inoffizieller Beichtvater, Coach oder Supervisor spielen kann. Wer noch einigermassen gesund ist, geniesst das grossartige Altersgeschenk der Freiheit, zu tun oder zu lassen, was man mag. Auch bei Klassen- und Jahrgängertreffen, im Alpenklub, im Freundes- und Bekanntenkreis trifft man immer wieder auf Menschen, die das einseitig negative Bild vom Alter Lügen strafen: so etwa der 87jährige Roger, der auf unseren SAC-Skitouren noch immer manchen Jüngeren überholt. Oder die 85jährige Malerin Annie, die trotz vielerlei Schmerzen und Gebrechen seit dem Tod ihres Mannes kreativ wie nie zuvor die Welt bereist. Mein Freund und früherer Chef Christian M., der im selben Alter Buch um Buch schreibt.

Ein einzigartiges Geschenk sind überhaupt die vielen Lebens- und auch Sterbensgeschichten, auf die man vom Beobachtungshochsitz des Alters aus zurückblicken kann. Nicht wenige haben bei aller Tragik zugleich etwas Tröstliches: so mein tiefgläubiger und bis zuletzt heiterer ehemaliger Patient und Kollege Dr. Z., der an einer chronischen Nervenkrankheit zugrunde ging und trotz heftigster Schmerzanfälle partout keine Analgetika wollte, um «seinen Anteil am unendlichen Leiden der Welt» zu leisten. Die Schwester meines Patenkinds F., die 37jährig eines fulminanten Darmkarzinoms wegen von Mann und zwei kleinen Kindern wegstarb und doch Angehörige und Mitpatienten auch noch im Sterbehaus wirksam tröstete. Ähnlich meine 56jährig verstorbene Cousine L., die trotz kaum erträglichen Krebsschmerzen bis zuletzt ihr Morphin immer wieder reduzierte, um ihre gesteigerte Sensibilität für Menschen, Farben und Töne zu bewahren.

\section{Schreckgespenst Alzheimer}

Das allerschlimmste ist für viele Menschen die Vorstellung, dereinst alzheimerkrank zu werden. Nur niemandem zur Last fallen! Nur nicht in entwürdigender Weise körperlich abhängig werden und zugleich unser kostbarstes Gut, nämlich Verstand und Gedächtnis, verlieren! - Aber gehört denn der körperliche wie psychische Abbau nicht genau gleich zum Leben wie der Aufbau? Und sind wir nicht von der Wiege bis zur Bahre in weit grösserem Ausmass von anderen Menschen abhängig - von ihren Dienstleistungen, ihrer Arbeit, ihrer Liebe und Anerkennung -, als wir dies gewöhnlich wahrhaben wollen? Der Mensch ist ein Herdentier; Kinder, Kranke und Alte bedürfen der Hilfe der Gemeinschaft.

Ich habe seinerzeit, wie schon erwähnt, das Innenleben von Alzheimerkranken - u.a. mit der psychoanalytischen Methode der freien Assoziation - zu erforschen versucht. Auch hier bin ich zu recht überraschenden Schlüssen gelangt: Diese Menschen sind meist keineswegs todunglücklich. Sie leben, von gelegentlichen Irritationen abgesehen, entweder heiter ganz im Hier und Jetzt - eigentlich genau das, was manche Esoteriker anstreben! - oder in einem traumartigen Nebel, in den sinnbefrachtete Fragmente des eigenen Lebens hell oder dunkel hineinragen. - «Lebensunwertes Leben»? Wer darf sich denn ein Urteil anmassen, wo Sinn und Wert doch primär von den Umständen, zumal der Beziehungssituation abhängen? Mir jedenfalls erscheint das Leben von Alzheimerpatienten, genauso wie das Leben anderer psychisch Kranker und Behinderter, als mindestens ebenso wertvoll wie dasjenige von - um es provokant auszudrücken - Schosshündchen oder Kanarienvögeln, um die wir ein vergleichsweise schockierendes Getue machen!

\section{Natürliches versus manipuliertes Sterben...}

Sehr beeindruckt hat mich auch die Geschichte des alten Knechts Louis aus unserem Feriendörfchen im Wallis, der, obwohl ganz gesund, eines Herbsts erklärte, nun komme sein letzter Winter, dann tatsächlich zunehmend schwächer wurde, bis er sich am Palmsonntag im Sonntagsstaat aufs Bett legte, Angehörige und Dorfgenossen kommen liess, einem nach dem anderen zum Abschied die Hand reichte und in der folgenden 
Nacht friedlich verstarb - ein «natürliches Sterben», das früher in diesem Bergtal nichts Aussergewöhnliches gewesen sein soll.

Was freilich heutzutage ein natürlicher Tod wäre, ist angesichts der fast unbegrenzten Manipulationsmöglichkeiten der modernen Medizin zwischen den Polen der monate- bis jahrelangen Verlängerung vegetativen Lebens (wie etwa bei Ariel Scharon) und der schleichend überhandnehmenden «ärztlichen Sterbehilfe» schwer zu sagen. Beide stürzen viele Menschen, mich selbst inbegriffen, in kaum lösbare Konflikte zwischen tiefverwurzelten Hemmungen vor der wohl gefährlichsten aller Grenzüberschreitungen und ebenso tiefem Mitgefühl mit qualvoll Leidenden. Schon hat das Lausanner CHUV als erstes Schweizer Spital einen besonderen Raum für den ärztlich assistierten Freitod eingerichtet. Bereits gibt es auch «Betagtenkurse» zu diesem Thema. Und allein in meiner näheren Umgebung habe ich in letzter Zeit zwei «Exit»-Sterbefälle miterlebt, die, obwohl rational gut verständlich, emotional doch einen seltsam bitteren Nachgeschmack hinterlassen. Wie so anders die für alle Beteiligen Frau und Exfrau, Kinder und Kindeskinder, Freunde und Bekannte - bei aller Tragik auch zutiefst bereichernde Geschichte meines alten
Bergfreunds M., der dank steter Stützung durch Familie und Umwelt fünf Jahre lang seinen progredienten Parkinson mit grosser Fassung ertragen, eine Zeitlang dann im Rollstuhl und kaum noch sprech- noch essfähig, einen «Exit»-Freitod ernsthaft ins Auge gefasst hatte und sich schliesslich doch noch dafür entschied, es ruhig «werden zu lassen». Bald darauf konnte er gelöst eines natürlichen Todes sterben.

\section{... und das Bild von der Eigernordwand}

«Niemand weiss, was die Eigernordwand ist, der sie nicht selbst durchklettert hat», sagte mir einst ein Bergführer. Ganz ähnlich ist es zweifellos mit Sterben und Tod. Wohl können und sollen wir uns zu beidem Gedanken machen. Wie man selbst aber in dieser steilen Wand einmal «klettern» wird, wenn es ernst gilt, das weiss im voraus keine/r.

\section{Literatur}

1 Ciompi L. Ärztliche Suizidbeihilfe und Ehrfurcht vor dem Leben. Schweiz Ärztezeitung. 2004; $85(3): 81-2$.

2 Neue Zürcher Zeitung Folio 4/2006: 20-4. 